\title{
The exponentiated logarithmic generated family of distributions and the evaluation of the confidence intervals by percentile bootstrap
}

\author{
Pedro Rafael Diniz Marinho ${ }^{\mathrm{a}}$, Gauss M. Cordeiro ${ }^{\mathrm{b}}$, Fernando Peña \\ Ramírez $^{\mathrm{b}}$, Morad Alizadeh $^{\mathrm{c}}$ and Marcelo Bourguignon ${ }^{\mathrm{d}}$

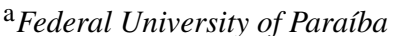 \\ ${ }^{\mathrm{b}}$ Federal University of Parnambuco \\ ${ }^{\mathrm{c}}$ Persian Gulf University of Bushehr \\ $\mathrm{d}_{\text {Federal University of Rio Grande do Norte }}$
}

\begin{abstract}
We study some mathematical properties of a new generator of continuous distributions with three additional parameters, called the exponentiated logarithmic generated family, to extend the normal, Weibull, gamma and Gumbel distributions, among other well-known models. Some special models are discussed. Many properties of this family are studied, some inference procedures developed and a simulation study performed to verify the adequacy of the estimators of the model parameters. We prove empirically the potentiality of the new family by means of two real data sets. The simulation study for different samples sizes assesses the performance of the maximum likelihood estimates obtained by the Swarm Optimization method. We also evaluate the performance of single and dual bootstrap methods in constructing interval estimates for the parameters. Because of the intensive simulations, we use parallel computing on a supercomputer.
\end{abstract}

\section{Introduction}

The modeling and analysis of lifetime distributions play an important role in a wide variety of practical fields such as biological and engineering sciences. However, in many practical situations, well-known continuous distributions do not provide an adequate fit. For example, if the data are asymmetric, the normal distribution will not be a good choice. So, several methods of introducing extra shape parameters to expand distributions have been studied.

The use of new generators of continuous distributions from classic ones has become very common in recent years. The beta-generated family was proposed by Eugene, Lee and Famoye (2002) and further discussed in Zografos and Balakrishnan (2009), who pioneered the gamma-generated family. More recently, Cordeiro and de Castro (2011) defined the Kumaraswamy-G family.

Key words and phrases. Bootstrap, generalized distribution, lifetime, logarithmic distribution, mixture.

Received May 2016; accepted November 2016. 
The chief motivation of the generalized distributions for modeling failure time data lies in its flexibility to model both monotonic and non-monotonic failure rates even though the baseline failure rate may be monotonic. The additional shape parameters aim to introduce skewness and to vary tail weights. Further, various distributions have been constructed by mixing some useful life distributions and analyzed them with respect to different characteristics.

In this paper, we propose a new method to add three parameters to a parent distribution with the hope that it yields a "better" fit in certain practical situations. Several properties are also investigated. Some inferential aspects of this family are studied in details, and four special cases are discussed. The new family of distributions shares an attractive interpretation (see Section 2). Further, two successful empirical applications show its flexibility and also motivate its applications.

This paper is unfolded as follows. In Section 2, we define the new family of distributions. Section 3 provides some special models. In Section 4, some of its basic mathematical properties are discussed. The formulas derived are manageable by using modern computer resources with analytic and numerical capabilities. In Section 5, the estimation of the model parameters is performed by the method of maximum likelihood. Section 6 deals with bootstrap percentile intervals. In Section 7, we apply a particle swarm optimization (PSO) method to estimate the parameters and present a Monte Carlo simulation experiment to evaluate the maximum likelihood estimates (MLEs). We also perform simulations to assess the use of bootstrap percentile (simple and double) for construction of confidence intervals for the model parameters. In Section 8, two applications based on real data sets are explored. Finally, concluding remarks are presented in Section 9.

\section{New generator}

The generator of continuous distributions presented is called the Exponentiated Logarithmic Generated (ELG) family with cumulative distribution function (cdf) given by

$$
F(x)=\left\{\frac{\log \left[1-a G(x, \xi)^{b}\right]}{\log (1-a)}\right\}^{c},
$$

where $a \in(0,1)$ (scale parameter), $b>0$ (shape parameter) and $c>0$ (shape parameter). The probability density function (pdf) obtained by differentiating $F(x)$ is given by

$$
f(x)=\frac{a b c[\log (1-a)]^{-c} g(x, \xi) G(x, \xi)^{b-1}\left\{\log \left[1-a G(x, \xi)^{b}\right]\right\}^{c-1}}{a G(x, \xi)^{b}-1} .
$$

For a given cdf $G(x ; \xi)$, denote by $f(x)$ the pdf of the Exponentiated Logarithmic Generated-G (ELG- $G$ ) distribution. 
Lemma 2.1. $f(x)$ given in (2) is a well-defined density function.

Proof. We observe that $f(x)$ is nonnegative. We prove that the integration over the support of the random variable is one. Consider the case when the support of $f(x)$ is $(-\infty, \infty)$. In this case, we have

$$
\begin{aligned}
\int_{-\infty}^{\infty} & f(x) d x \\
& =\int_{-\infty}^{\infty} \frac{-a b c[\log (1-a)]^{-c} g(x, \xi) G(x, \xi)^{b-1}\left\{\log \left[1-a G(x, \xi)^{b}\right]\right\}^{c-1}}{1-a G(x)^{b}} d x \\
& =\frac{-c}{[\log (1-a)]^{c}} \int_{0}^{a} \frac{[\log (1-u)]^{c-1}}{1-u} d u=1
\end{aligned}
$$

Henceforth, we denote by $X$ a random variable having pdf (2). For simulating data from the ELG- $G$ distribution, let $u \sim U(0,1)$. The solution of non-linear equation

$$
x_{u}=Q_{G}\left\{\left[\frac{1}{a}\left(1-[1-a]^{u^{\frac{1}{c}}}\right)\right]^{\frac{1}{b}}\right\}
$$

has cdf (1), where $Q_{G}=G^{-1}(\cdot)$ is the quantile function (qf) of $G$.

For an interpretation of this family, we consider a parallel system with $N$ independent components, where $N$ is a random variable with probability mass function (pmf)

$$
P(N=n)=\frac{-1}{\log (1-a)} \frac{a^{n}}{n}, \quad 0<a<1, n \in \mathbb{N} .
$$

Suppose that $Z_{1}, \ldots, Z_{N}$ are independent identically distributed (i.i.d.) random variables with common cdf $G(x)^{b}$. Then, $M_{N}=\max \left(X_{1}, \ldots, X_{N}\right)$ represents the lifetime of the system and

$$
\begin{aligned}
\Pi_{M_{N}}(x) & =\sum_{n=1}^{\infty} P\left(M_{N} \leq x \mid N=n\right) P(N=n) \\
& =\sum_{n=1}^{\infty} \frac{-1}{\log (1-a)} \frac{a^{n}}{n}\left[G(x)^{b}\right]^{n}=\frac{\log \left[1-a G(x)^{b}\right]}{\log (1-a)} .
\end{aligned}
$$

For $c$ positive integer, we consider a system formed by independent components having the cdf above. Suppose the system fails if all $c$ components fail. Then, the cdf of $X$ is given by (1).

Remark 2.1. The following properties use Stirling polynomials. We consider the basic formula for the Stirling polynomial proposed by Ward (1934, pp. 87-95). The notation for the Stirling polynomial adopted is $\psi_{n-1}(x)$ in accordance with 
the notation presented by Ward (1934, p. 87). The Stirling polynomial is defined by

$$
\begin{aligned}
\psi_{n-1}(x)= & \frac{(-1)^{n-1}}{(n+1) !}\left[H_{n}^{n-1}-\frac{x+2}{n+2} H_{n}^{n-2}+\frac{(x+2)(x+3)}{(n+2)(n+3)} H_{n}^{n-3}\right. \\
& \left.-\cdots+(-1)^{n-1} \frac{(x+2)(x+3) \cdots(x+n)}{(n+2)(n+3) \cdots(2 n)} H_{n}^{0}\right],
\end{aligned}
$$

where the $H_{n}^{m}$ 's are positive integers defined recursively by $H_{n+1}^{m}=(2 n+1-$ m) $H_{n}^{m}+(n-m+1) H_{n}^{m-1}$, and $H_{0}^{0}=1, H_{n+1}^{0}=1 \times 3 \times 5 \times \cdots \times(2 n+1)$ and $H_{n+1}^{n}=1$.

To avoid recursion in equation (4), the quantities $H_{n+1}^{m}$ can follow the Stirling polynomial given in http://mathworld.wolfram.com/StirlingPolynomial.html. Let

$$
S_{n}(m)=\frac{(-1)^{n}}{\left(\begin{array}{c}
m \\
n
\end{array}\right)} s(m+1, m-n+1)
$$

where $m \geq n$, and $s(n, m)$ is the Stirling number of the first kind defined by Roman (1984, p. 129). We can obtain $s(n, m)$ from the Stirling numbers of the second kind defined by

$$
S(n, m)=\frac{1}{m !} \sum_{i=0}^{m}(-1)^{i}\left(\begin{array}{c}
m \\
i
\end{array}\right)(n-i)^{n}
$$

Based on (6), we have

$$
s(n, m)=\sum_{k=0}^{n-m}(-1)^{k}\left(\begin{array}{c}
k+n-1 \\
k+n-m
\end{array}\right)\left(\begin{array}{c}
2 n-m \\
n-k-m
\end{array}\right) S(k-m+n, k) .
$$

More details about equation (7) and the relationship between first order Stirling numbers and second order Stirling numbers can be obtained in http://mathworld. wolfram.com/StirlingNumberoftheFirstKind.html.

Thus, according with Castellares and Lemonte $(2015$, p. 2$)$, we have $\psi_{n-1}(x)=$ $S_{n}(x) /[n !(x+1)]$. So, we can determine $\psi_{n-1}(x)$ without explicitly the values of $H_{n}^{m}$. Castellares and Lemonte $(2015$, p. 5) developed scripts in the R language to evaluate the quantities $H_{m}^{n}$ and $\psi_{n}(\cdot)$. We give in the Appendix an implemented function in the Julia programming language (Bezanson et al., 2012) to evaluate the Stirling polynomial at the point $x$ defined by (4) and functions of the Stirling numbers of first and second order given by equations (5) and (6), respectively.

Proposition 2.2. Let $\left[-\frac{\log (1-z)}{z}\right]^{\delta}=1+\delta z \sum_{n=0}^{\infty} \psi_{n}(n+\delta) z^{n}$, where $\delta \in \mathbb{R}$ and $|z|<1$. This expansion is absolutely convergent.

Proof. The proof is given by Flajonet and Odlyzko (1990) and Flajonet and Sedgewick (2009) (see Theorem VI.2, p. 385). 
If we know an expansion for $\left[-\frac{\log (1-z)}{z}\right]^{\delta}$ for $\delta>0$ and $|z|<1$, and that this expansion converges absolutely, it is easy to obtain another expansion for $[-\log (1-z)]^{\delta}$ that is also absolutely convergent. Thus, it follows that

$$
[-\log (1-z)]^{\delta}=z^{\delta}+\delta \sum_{n=0}^{\infty} \psi_{n}(n+\delta) z^{\delta+n+1}
$$

Proposition 2.3. Based on the assumptions below, it is possible to obtain equation (8). Let

$$
[-\log (1-z)]^{\delta}=z^{\delta} \sum_{m=0}^{\infty} \rho_{m}(\delta) z^{m}
$$

where $\delta \in \mathbb{R},|z|<1, \rho_{0}(\delta)=1, \rho_{m}(\delta)=\delta \psi_{m-1}(m+\delta-1)$ for $m \geq 1$ and $\psi_{m}(\cdot)$ is a Stirling polynomial.

Proof. Note that

$$
\begin{aligned}
{[-\log (1-z)]^{\delta} } & =z^{\delta} \sum_{m=0}^{\infty} \rho_{m}(\delta) z^{m}=z^{\delta}\left[\rho_{0}(\delta) z^{0}+\sum_{m=1}^{\infty} \rho_{m}(\delta) z^{m}\right] \\
& =z^{\delta}+\sum_{m=1}^{\infty} \rho_{m}(\delta) z^{m+\delta}=z^{\delta}+\delta \sum_{m=0}^{\infty} \psi_{m}(m+\delta) z^{\delta+m+1}
\end{aligned}
$$

Thus, it follows that the cdf $F(x)$ can be expressed as a mixture of exponentiated-G $(\exp -\mathrm{G})$ cdfs with power parameters $(m+c) b$, say exp-G $([m+c] b)$. We have

$$
F(x)=\sum_{m=0}^{\infty} b_{m} H_{(m+c) b}(x),
$$

where $H_{(m+c) b}(x)$ is the cdf of the exp-G([m+c]b) distribution and $b_{m}$ (for $m \geq 0$ ) are constants defined by

$$
b_{m}=\frac{\rho_{m}(c) a^{m+c}}{[-\log (1-a)]^{c}} .
$$

By differentiating (10), we obtain

$$
f(x)=\sum_{m=0}^{\infty} b_{m} h_{(m+c) b},
$$

where $h_{(m+c) b}(x)$ is the exp-G density with power parameter $(m+c) b$. We have $\sum_{m=0}^{\infty} b_{m}=1$. Figure 1 displays the convergence of $\sum_{m=0}^{n} b_{m}$ with $n=$ $1,2, \ldots, 15, a=0.5$ and $c=1.2$. 


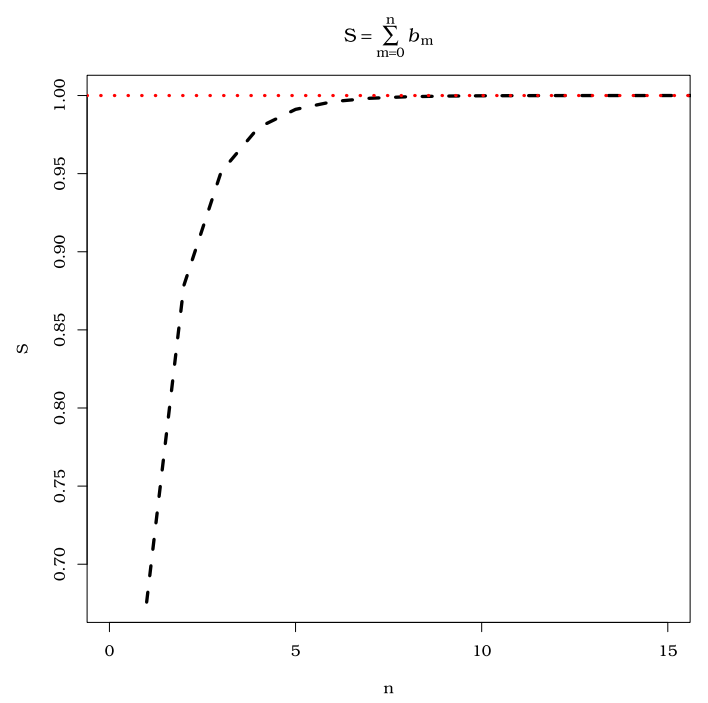

Figure 1 Sums of the constants of the linear combination in equation (10).

\section{Special distributions}

Four of the many distributions obtained as special models of the ELG-G family are given in this section. We consider the baseline distributions: normal, Weibull, gamma and log-logistic. The last three distributions are largely used in survival analysis. In the following, we shall provide the pdf and some plots of the hazard rate function (hrf) for each of these four cases. Clearly, $a, b$ and $c$ are the generatorG parameters.

\subsection{ELG-normal}

In applied statistics, the normal distribution is the most popular model of the sample size is large, it can serve as an approximate distribution to other models. The ELG-normal distribution is defined from (1) by taking $G(x)$ and $g(x)$ to be the cdf and pdf of the normal $\mathrm{N}\left(\mu, \sigma^{2}\right)$ distribution. Its density function is given by

$$
f_{G N}(x)=\frac{-a b c[\log (1-a)]^{-c} \Phi\left(\frac{x-\mu}{\sigma}\right)^{b-1} \phi\left(\frac{x-\mu}{\sigma}\right)\left[\log \left(1-a \Phi\left(\frac{x-\mu}{\sigma}\right)^{b}\right)\right]^{c-1}}{\sigma\left\{1-a \Phi\left(\frac{x-\mu}{\sigma}\right)^{b}\right\}},
$$

where $x \in \mathbb{R}, \mu \in \mathbb{R}$ is a location parameter, $\sigma>0$ and $a \in(0,1)$ are the scale parameters, $b, c$ are the shape parameters, and $\phi(\cdot)$ and $\Phi(\cdot)$ are the pdf and cdf of the standard normal distribution, respectively.

Plots of the ELG-normal density for some parameter values are displayed in Figure 2. It is evident that this distribution is much more flexible than the normal distribution. 

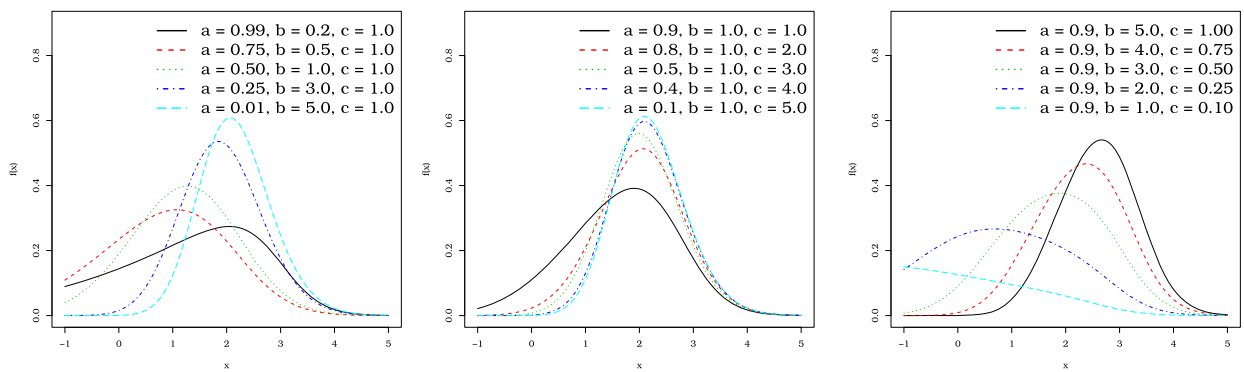

Figure 2 The ELG-normal pdffor $\mu=1, \sigma=1$ and some values of $a, b$ and $c$.
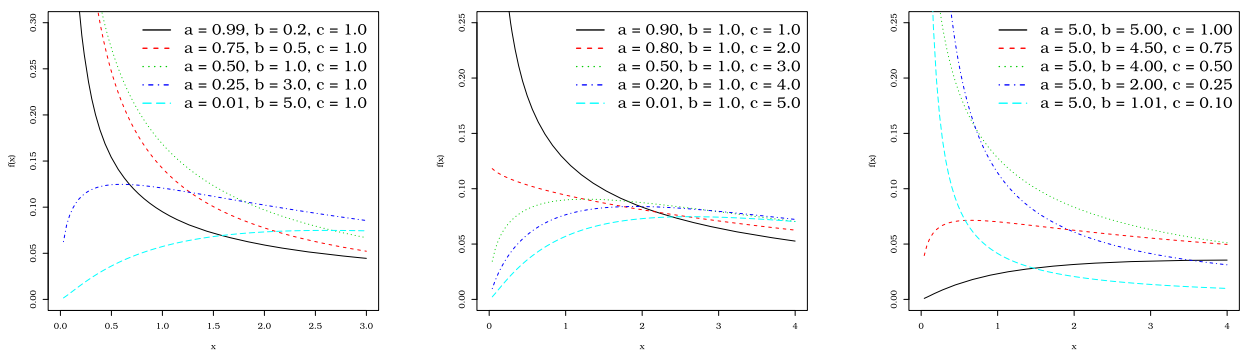

Figure 3 The ELG-Weibull pdffor $\alpha=0.5, \beta=2.0$ and some values of $a, b$ and $c$.

\subsection{ELG-Weibull}

The Weibull distribution is a very popular model due to its large applicability in survival analysis. Let $G(x)$ be the Weibull cdf with scale parameter $\beta>0$ and shape parameter $\alpha>0$, say $G(x)=1-\exp \left\{-(\beta x)^{\alpha}\right\}$, for $x>0$. The ELGWeibull density function is obtained from (2) as

$$
\begin{aligned}
& f_{\mathrm{ELG}-\mathrm{W}}(x) \\
& \quad=\frac{-a b c\left[1-\mathrm{e}^{-(x \beta)^{\alpha}}\right]^{b-1} \alpha \beta^{\alpha} x^{\alpha-1} \mathrm{e}^{-(x \beta)^{\alpha}}\left\{\log \left\{1-a\left[1-\mathrm{e}^{-(x \beta)^{\alpha}}\right]^{b}\right\}\right\}^{c-1}}{[\log (1-a)]^{c}\left\{1-a\left[1-\mathrm{e}^{-(x \beta)^{\alpha}}\right]^{b}\right\}} .
\end{aligned}
$$

Plots of this density function for selected parameter values are given in Figure 3.

Figure 4 provides some plots of the ELG-Weibull hrf for selected parameter values.

\subsection{ELG-gamma}

Another distribution that is frequently used in survival analysis is the gamma distribution. Taking $G(x)$ to be gamma cdf with shape $\alpha>0$ and scale $\beta>0$, say $G(x)=\gamma(\alpha, x / \beta) / \Gamma(\alpha)$, where $\Gamma(\alpha)=\int_{0}^{\infty} t^{\alpha-1} \mathrm{e}^{-t} d t$ denotes the gamma function, and $\gamma(\alpha, z)=\int_{0}^{z} t^{\alpha-1} \mathrm{e}^{-t} d t$ denotes the incomplete gamma function, the 

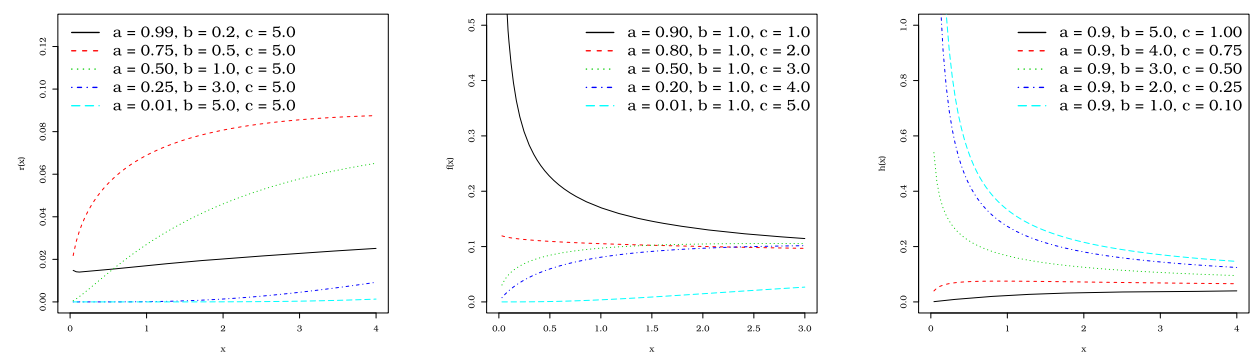

Figure 4 The ELG-Weibull hrffor $\alpha=0.5, \beta=2.0$ and some values of $a, b$ and $c$.
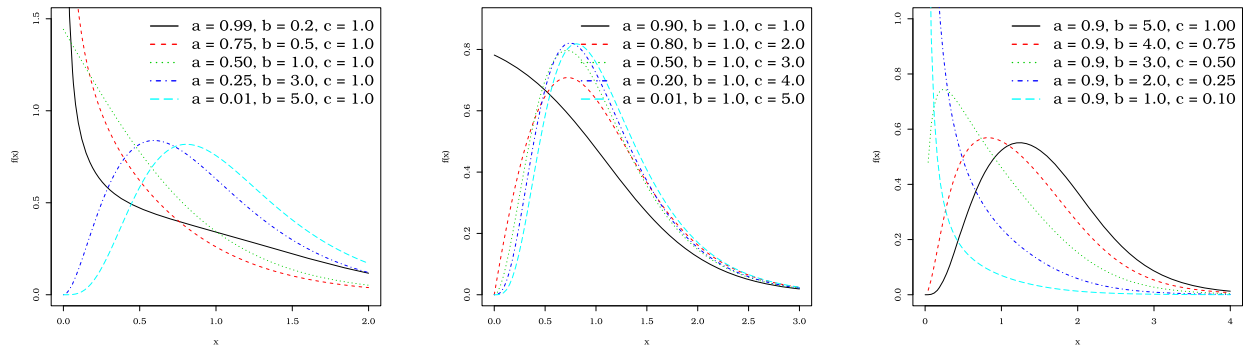

Figure 5 The ELG-gamma pdffor $\alpha=1.0, \beta=2.0$ and some values of $a, b$ and $c$.
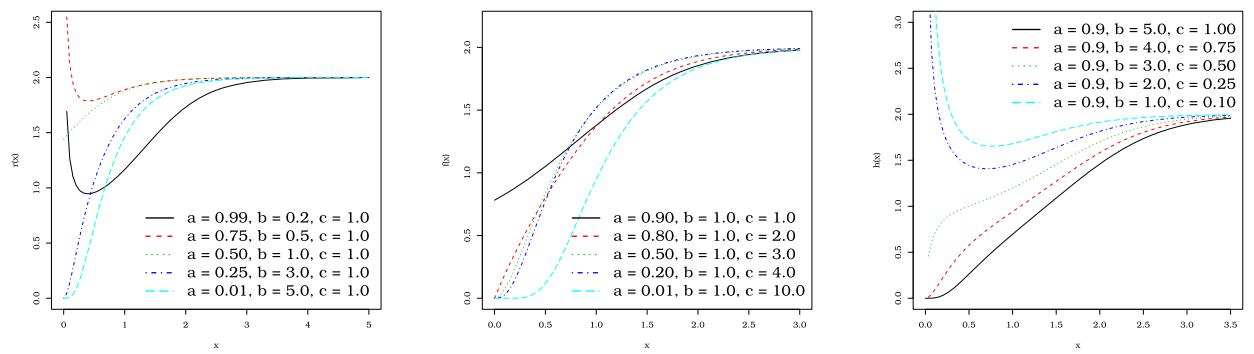

Figure 6 The ELG-gamma hrf for $\alpha=1.0, \beta=2.0$ and some values of $a, b$ and $c$.

ELG-gamma density (for $x>0$ ) becomes

$$
f_{\mathrm{ELG}-\mathrm{Ga}}(x)=\frac{-a b c x^{\alpha-1} \mathrm{e}^{-x / \beta}\left[\frac{\gamma(\alpha, x / \beta)}{\Gamma(\alpha)}\right]^{b-1}\left\{\log \left\{1-a\left[\frac{\gamma(\alpha, x / \beta)}{\Gamma(\alpha)}\right]^{b}\right\}\right\}^{c-1}}{\beta^{\alpha} \Gamma(\alpha)[\log (1-a)]^{c}\left\{1-a\left[\frac{\gamma(\alpha, x / \beta)}{\Gamma(\alpha)}\right]^{b}\right\}} .
$$

Figure 5 displays some plots of the ELG-gamma density. They reveal that this distribution has great flexibility.

Plots of the ELG-gamma hrf for selected parameter values are displayed in Figure 6 . The monotonically increasing and bathtub shapes are evident. 


\subsection{ELG-log-logistic}

Consider the log-logistic distribution with scale parameter $\alpha>0$ and shape parameter $\beta>0$, where the pdf and cdf (for $x>0$ ) are

$$
g(x)=\frac{\beta}{\alpha^{\beta}} x^{\beta-1}\left[1+\left(\frac{x}{\alpha}\right)\right]^{-2} \text { and } G(x)=1-\left[1+\left(\frac{x}{\alpha}\right)^{\beta}\right]^{-1} .
$$

Inserting these expressions into (2) gives the ELG-log-logistic pdf

$$
\begin{aligned}
& f_{\mathrm{ELG}-\mathrm{LL}}(x) \\
& \quad=\frac{-a b c \beta x^{\beta-1}\left[1-\frac{1}{1+(x / \alpha)^{\beta}}\right]^{b-1}\left\{\log \left\{1-a\left[1-\frac{1}{1+(x / \alpha)^{\beta}}\right]^{b}\right\}\right\}^{c-1}}{\alpha^{\beta}[\log (1-a)]^{c}\left(1+\frac{x}{\alpha}\right)^{2}\left\{1-a\left[1-\frac{1}{1+(x / \alpha)^{\beta}}\right]^{b}\right\}} .
\end{aligned}
$$

Figure 7 provides plots shapes of the ELG-log-logistic pdf for selected parameters values.

A random variable with density (14) is denoted by $X \sim \operatorname{ELGLL}(a, b, c, \alpha, \beta)$. For $\alpha=2$ and $\beta=2$, possible shapes for the hrf of $X$ are displayed in Figure 8.
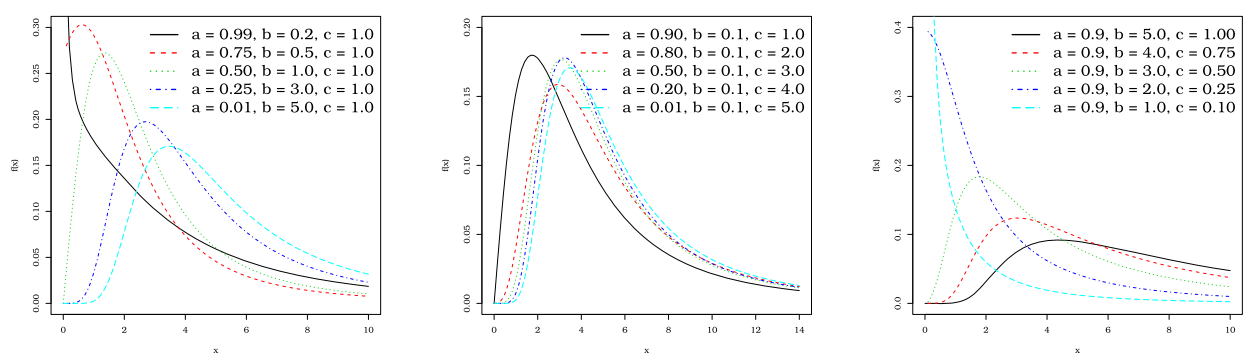

Figure 7 The ELG-log-logistic pdffor $\alpha=2.0, \beta=2.0$ and some values of $a, b$ and $c$.
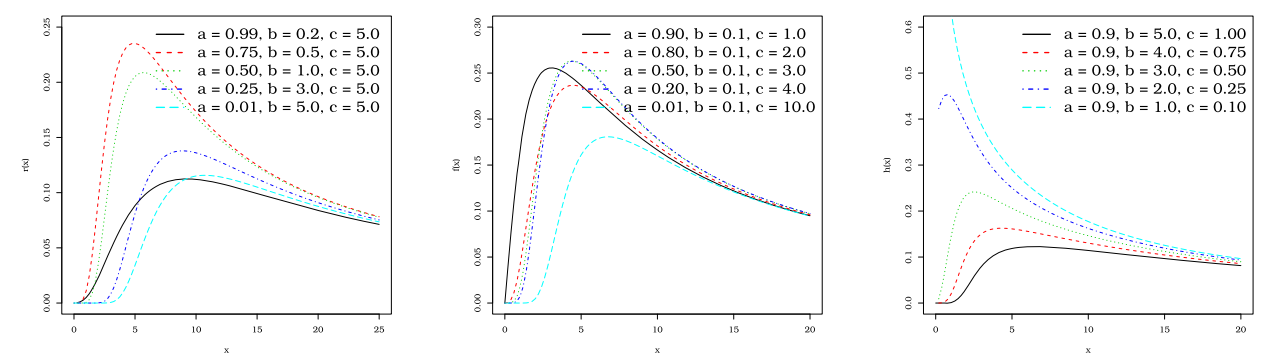

Figure 8 The ELG-log-logistic hrffor $\alpha=2.0, \beta=2.0$ and some values of $a, b$ and $c$. 


\section{Mathematical properties}

\subsection{Moments and generating function}

We assume that $Y$ is a random variable having the baseline $\operatorname{cdf} G(x)$. The moments of $X$ can be obtained from the $(r, k)$ th probability weighted moment (PWM) of $Y$ defined by

$$
\omega_{r, k}=\mathrm{E}\left[Y^{r} G(Y)^{k}\right]=\int_{-\infty}^{\infty} x^{r} G(x)^{k} g(x) d x,
$$

which can be evaluated at least numerically for any baseline qf. We can write from equation (10)

$$
\mu_{r}^{\prime}=\mathrm{E}\left(X^{r}\right)=\sum_{m=0}^{\infty}[(m+c) b] b_{m} \omega_{r,(m+c) b-1} .
$$

The $n$th incomplete moment of $X$ is defined as $m_{n}(y)=\int_{-\infty}^{y} x^{n} f(x) d x$. Then, it can be expressed as

$$
m_{n}(y)=\sum_{m=0}^{\infty} b_{m} \int_{0}^{G(y ; \boldsymbol{\xi})} Q_{G}(u)^{n} u^{(m+c) b} d u .
$$

The integral in (15) can be evaluated at least numerically for most baseline distributions.

We provide two formulae for the moment generating function (mgf) $M(s)=$ $\mathrm{E}\left(\mathrm{e}^{s X}\right)$ of $X$. The first formula for $M(s)$ comes from equation (10) as

$$
M(s)=\sum_{m=0}^{\infty} b_{m} M_{(m+c) b}(s),
$$

where $M_{(m+c) b}(s)$ is the exp-G generating function with power parameter $(m+$ c) $b$.

Equation (16) can also be expressed as

$$
M(s)=\sum_{m=0}^{\infty}[(m+c) b] b_{m} \rho_{(m+c) b}(s),
$$

where the quantity $\rho_{k}(s)=\int_{0}^{1} \exp \left[s Q_{G}(u)\right] u^{(m+c) b} d u$ can be evaluated numerically.

\subsection{Order statistics}

Suppose $X_{1}, \ldots, X_{n}$ is a random sample from any ELG-G distribution. Let $X_{i: n}$ denote the $i$ th order statistic. The pdf of $X_{i: n}$ can be expressed as

$$
f_{i: n}(x)=K f(x) F^{i-1}(x)\{1-F(x)\}^{n-i},
$$

where $K=n ! /[(i-1) !(n-i) !]$. 
The density function of the $i$ th order statistic of any ELG-G distribution can be expressed as

$$
\begin{aligned}
f_{i: n}(x)= & K \sum_{j=0}^{n-i}(-1)^{j}\left(\begin{array}{c}
n-i \\
j
\end{array}\right) f(x) F(x)^{j+i-1} \\
= & \frac{K a b c}{[-\log (1-a)]^{c}} \sum_{j=0}^{n-i}(-1)^{j}\left(\begin{array}{c}
n-i \\
j
\end{array}\right) \\
& \times \frac{g(x) G(x)^{b-1}\left\{-\log \left[1-a G(x)^{b}\right]\right\}^{c(i+j)-1}}{1-a G(x)^{b}} \\
= & \frac{K a b c}{[-\log (1-a)]^{c}} \sum_{j=0}^{n-i} \sum_{k=0}^{\infty}(-1)^{j} a^{k}\left(\begin{array}{c}
n-i \\
j
\end{array}\right) \\
& \times \frac{g(x) G(x)^{b(k+1)-1}\left\{-\log \left[1-a G(x)^{b}\right]\right\}^{c(i+j)-1}}{1-a G(x)^{b}} .
\end{aligned}
$$

Using equation (9), we can write

$$
\begin{aligned}
& \left\{-\log \left[1-a G(x)^{b}\right]\right\}^{c(i+j)-1} \\
& \quad=\left[a G(x)^{b}\right]^{c(i+j-1)} \sum_{m=0}^{\infty} \rho_{m}(c(i+j)-1)\left[a G(x)^{b}\right]^{m}
\end{aligned}
$$

and then

$$
f_{i: n}(x)=\sum_{k, m=0}^{\infty} v_{k, m}^{(i)} h_{b(k+m+c i+c j)}(x)
$$

where

$$
v_{k, m}^{(i)}=\frac{K b c}{[-\log (1-a)]^{c}} \sum_{j=0}^{n-i} \frac{(-1)^{j}\left(\begin{array}{c}
n-i \\
j
\end{array}\right) a^{k+m+c(i+j)} \rho_{m}(c(i+j)-1)}{[b(k+m+c i+c j+1)]}
$$

and $h_{b(k+m+c i+c j)}(x)$ denotes the exp-G density function with parameter $b(k+$ $m+c i+c j)$.

We can obtain the ordinary and incomplete moments, generating function and mean deviations of the ELG-G order statistics from equation (19) and those properties of the exp-G model.

\section{Maximum likelihood estimation}

In this section, we determine the maximum likelihood estimates (MLEs) of the parameters of the new family of distributions. Let $x_{1}, \ldots, x_{n}$ be the observed values 
from the ELG-G distribution with the $p \times 1$ parameter vector $\boldsymbol{\theta}=(a, b, c, \boldsymbol{\xi})^{\top}$. We determine the MLEs of the parameters in $\boldsymbol{\theta}$ from complete samples only. The total $\log$-likelihood function for $\boldsymbol{\theta}$ is given by

$$
\begin{aligned}
\ell(\boldsymbol{\theta})= & n \log (a b c)-n c \log [-\log (1-a)] \\
& +\sum_{i=1}^{n} \log \left[g\left(x_{i} ; \boldsymbol{\xi}\right)\right]+(b-1) \sum_{i=1}^{n} G\left(x_{i} ; \boldsymbol{\xi}\right) \\
& +(c-1) \sum_{i=1}^{n} \log \left\{-\log \left[1-a G\left(x_{i} ; \boldsymbol{\xi}\right)^{b}\right]\right\} \\
& -\sum_{i=1}^{n} \log \left[1-a G\left(x_{i} ; \boldsymbol{\xi}\right)^{b}\right] .
\end{aligned}
$$

The components of the score function $U(\boldsymbol{\theta})=\left(U_{a}, U_{b}, U_{c}, U_{\xi}\right)^{\top}$ are

$$
\begin{aligned}
& U_{a}=\frac{n}{a}+\frac{n c}{(1-a) \log (1-a)}+\sum_{i=1}^{n} t_{i}^{(a)}+(c-1) \sum_{i=1}^{n} \frac{t_{i}^{(a)}}{t_{i}}, \\
& U_{b}=\frac{n}{b}+\sum_{i=1}^{n} \log \left[G\left(x_{i} ; \xi\right)\right]+\sum_{i=1}^{n} t_{i}^{(b)}+(c-1) \sum_{i=1}^{n} \frac{t_{i}^{(b)}}{t_{i}}, \\
& U_{c}=\frac{n}{c}-n \log [-\log (1-a)]+\sum_{i=1}^{n} \log \left(t_{i}\right)
\end{aligned}
$$

and

$$
U_{\xi_{k}}=\sum_{i=1}^{n} \frac{g\left(x_{i} ; \boldsymbol{\xi}\right)^{(\xi)}}{g\left(x_{i} ; \boldsymbol{\xi}\right)}+(b-1) \sum_{i=1}^{n} \frac{g\left(x_{i} ; \boldsymbol{\xi}\right)^{(\xi)}}{g\left(x_{i} ; \xi\right)}+\sum_{i=1}^{n} t_{i}^{(\xi)}+(c-1) \sum_{i=1}^{n} \frac{t_{i}^{(\xi)}}{t_{i}},
$$

where

$$
\begin{aligned}
t_{i} & =-\log \left[1-a G\left(x_{i} ; \xi\right)^{b}\right], \quad t_{i}^{(a)}=\frac{G\left(x_{i} ; \xi\right)^{b}}{1-a G\left(x_{i} ; \xi\right)^{b}}, \\
t_{i}^{(b)} & =\frac{a G\left(x_{i} ; \xi\right)^{b} \log \left[G\left(x_{i} ; \xi\right)\right]}{1-a G\left(x_{i} ; \xi\right)^{b}}, \\
g\left(x_{i} ; \xi\right)^{(\xi)} & =\frac{\partial g\left(x_{i} ; \xi\right)}{\partial \xi}, \quad G\left(x_{i} ; \xi\right)^{(\xi)}=\frac{\partial G\left(x_{i} ; \xi\right)}{\partial \xi}, \\
t_{i}^{(\xi)} & =\frac{a b G\left(x_{i} ; \xi\right)^{(\xi)} G\left(x_{i} ; \xi\right)^{b-1}}{1-a G\left(x_{i} ; \xi\right)^{b}} .
\end{aligned}
$$

Setting $U_{a}, U_{b}, U_{c}$ and $U_{\xi}$ equal to zero and solving the equations simultaneously yields the MLE $\hat{\boldsymbol{\theta}}=(\hat{a}, \hat{b}, \hat{c}, \hat{\boldsymbol{\xi}})^{\top}$ of $\boldsymbol{\theta}=(a, b, c, \boldsymbol{\xi})^{\top}$. These equations 
cannot be solved analytically and statistical software can be used to solve them numerically using iterative methods such as the Newton-Raphson type algorithms.

Since Fisher's information matrix is not available, the standard errors can be obtained as the square roots of the elements in the diagonal of the inverse of the negative of the observed information matrix of the log-likelihood calculated at the MLEs. The observed information matrix is given by

$$
J(\boldsymbol{\theta})=-\left(\begin{array}{ccccc}
U_{a a} & U_{a b} & U_{a c} & \mid & U_{a \xi}^{\top} \\
U_{b a} & U_{b b} & U_{b c} & \mid & U_{b \boldsymbol{\xi}}^{\top} \\
U_{c a} & U_{c b} & U_{c c} & \mid & U_{c \boldsymbol{\xi}}^{\top} \\
-- & -- & -- & -- & -- \\
U_{a \xi} & U_{b \xi} & U_{c \xi} & \mid & U_{\xi \xi}
\end{array}\right),
$$

whose elements are

$$
\begin{aligned}
& U_{a a}=-\frac{n}{a^{2}}+\frac{n c}{(1-a)^{2} \log (1-a)}+\frac{n c}{(1-a) \log (1-a)^{2}} \\
& +\sum_{i=1}^{n} t_{i}^{(a a)}+(c-1) \sum_{i=1}^{n} \frac{t_{i}^{(a a)} t_{i}-\left[t_{i}^{(a)}\right]^{2}}{t_{i}^{2}}, \\
& U_{a b}=\sum_{i=1}^{n} t_{i}^{(a b)}+(c-1) \sum_{i=1}^{n} \frac{t_{i}^{(a b)} t_{i}-t_{i}^{(a)} t_{i}^{(b)}}{t_{i}^{2}}, \\
& U_{a c}=\frac{n}{(1-a) \log (1-a)}+\sum_{i=1}^{n} \frac{t_{i}^{(a)}}{t_{i}}, \\
& U_{a \xi_{k}}=\sum_{i=1}^{n} t_{i}^{(a \xi)}+(c-1) \sum_{i=1}^{n} \frac{t_{i}^{(a \xi)} t_{i}-t_{i}^{(a)} t_{i}^{(\xi)}}{t_{i}^{2}}, \\
& U_{b b}=\frac{n}{b^{2}}+\sum_{i=1}^{n} t_{i}^{(b b)}+(c-1) \sum_{i=1}^{n} \frac{t_{i}^{(b b)} t_{i}-\left[t_{i}^{(b)}\right]^{2}}{t_{i}^{2}} \\
& U_{b c}=\sum_{i=1}^{n} \frac{t_{i}^{(b)}}{t_{i}} \\
& U_{b \boldsymbol{\xi}_{k}}=\sum_{i=1}^{n} \frac{G\left(x_{i} ; \boldsymbol{\xi}\right)^{(\xi)}}{G\left(x_{i} ; \xi\right)}+\sum_{i=1}^{n} t_{i}^{(b \xi)}+(c-1) \sum_{i=1}^{n} \frac{t_{i}^{(b \xi)} t_{i}-t_{i}^{(b)} t_{i}^{(\xi)}}{t_{i}^{2}} \\
& U_{c c}=-\frac{n}{c^{2}} \text {, } \\
& U_{\xi_{k} \xi_{l}}=\sum_{i=1}^{n} \frac{g\left(x_{i} ; \xi\right)^{(\xi \xi)}-\left[g\left(x_{i} ; \xi\right)^{(\xi)}\right]^{2}}{g\left(x_{i} ; \xi\right)^{2}}
\end{aligned}
$$




$$
\begin{aligned}
& +(b-1) \sum_{i=1}^{n} \frac{G\left(x_{i} ; \xi\right)^{(\xi \xi)}-\left[G\left(x_{i} ; \xi\right)^{(\xi)}\right]^{2}}{G\left(x_{i} ; \xi\right)^{2}} \\
& +\sum_{i=1}^{n} t_{i}^{(\xi \xi)}+(c-1) \sum_{i=1}^{n} \frac{t_{i}^{(\xi \xi)} t_{i}-\left[t_{i}^{(\xi)}\right]^{2}}{t_{i}^{2}} \\
U_{c \xi_{k}}= & \sum_{i=1}^{n} \frac{t_{i}^{(\xi)}}{t_{i}},
\end{aligned}
$$

where

$$
\begin{aligned}
& t_{i}^{(a a)}=\left\{\frac{G\left(x_{i} ; \xi\right)^{b}}{1-a G\left(x_{i} ; \xi\right)^{b}}\right\}^{2}, \quad t_{i}^{(a b)}=\frac{G\left(x_{i} ; \xi\right)^{b} \log \left[G\left(x_{i} ; \boldsymbol{\xi}\right)\right]}{\left[1-a G\left(x_{i} ; \xi\right)^{b}\right]^{2}}, \\
& t_{i}^{(b b)}=\left\{\frac{G\left(x_{i} ; \xi\right)^{b} \log \left[G\left(x_{i} ; \xi\right)\right]}{1-a G\left(x_{i} ; \xi\right)^{b}}\right\}^{2}, \quad t_{i}^{(a \xi)}=\frac{b G\left(x_{i} ; \xi\right)^{(\xi)} G\left(x_{i} ; \xi\right)^{b-1}}{\left[1-a G\left(x_{i} ; \xi\right)^{b}\right]^{2}} \\
& t_{i}^{(b \boldsymbol{\xi})}=a G\left(x_{i} ; \boldsymbol{\xi}\right)^{(\xi)}\left\{\left[\frac{G\left(x_{i} ; \xi\right)^{b}}{1-a G\left(x_{i} ; \xi\right)^{b}}\right]^{2}+\frac{G\left(x_{i} ; \xi\right)^{b-1}}{1-a G\left(x_{i} ; \xi\right)^{b}}\right\}, \\
& g\left(x_{i} ; \boldsymbol{\xi}\right)^{(\xi \xi)}=\frac{\partial^{2} g\left(x_{i} ; \boldsymbol{\xi}\right)}{\partial \xi^{2}}, \quad G\left(x_{i} ; \xi\right)^{(\xi \xi)}=\frac{\partial^{2} G\left(x_{i} ; \xi\right)}{\partial \xi^{2}}, \\
& t_{i}^{(\xi \xi)}=\frac{a b G\left(x_{i} ; \boldsymbol{\xi}\right)^{(\xi \xi)} G\left(x_{i} ; \xi\right)^{b-1}}{1-a G\left(x_{i} ; \xi\right)^{b}}+\frac{a b(b-1)\left[G\left(x_{i} ; \xi\right)^{(\xi)}\right]^{2} G\left(x_{i} ; \xi\right)^{b-2}}{1-a G\left(x_{i} ; \xi\right)^{b}} \\
& +\left\{\frac{a b G\left(x_{i} ; \xi\right)^{(\xi)} G\left(x_{i} ; \xi\right)^{b-1}}{1-a G\left(x_{i} ; \xi\right)^{b}}\right\}^{2} .
\end{aligned}
$$

Besides estimation of the model parameters, hypotheses tests can be taken into account. We can compute the maximum values of the unrestricted and restricted log-likelihoods to obtain the likelihood ratio (LR) statistics for testing some submodels of the ELG-G distribution. Let $\boldsymbol{\theta}=\left(\boldsymbol{\theta}_{1}^{\top}, \boldsymbol{\theta}_{2}^{\top}\right)^{\top}$, where $\boldsymbol{\theta}_{1}$ and $\boldsymbol{\theta}_{2}$ are disjoint subsets of $\boldsymbol{\theta}$. The LR statistic for testing the null hypothesis $H_{0}: \boldsymbol{\theta}_{1}=\boldsymbol{\theta}_{1}^{(0)}$ versus the alternative hypothesis $H_{1}: \boldsymbol{\theta}_{1} \neq \boldsymbol{\theta}_{1}^{(0)}$ is given by $w=2\{\ell(\hat{\boldsymbol{\theta}})-\ell(\tilde{\boldsymbol{\theta}})\}$, where $\hat{\boldsymbol{\theta}}$ and $\tilde{\boldsymbol{\theta}}$ are the MLEs under the alternative and null hypotheses, respectively. The statistic $w$ is asymptotically distributed as $\chi_{k}^{2}$, where $k$ is the dimension of the subset $\boldsymbol{\theta}_{1}$ of interest.

\section{Bootstrap confidence intervals}

The bootstrap method was pioneered in 1979 by Efron (1979), who revitalized the jackknife resampling methodology and established a new area of research. The bootstrap methods present two approaches: the parametric bootstrap and nonparametric bootstrap. Parametric bootstrap refers to the case where the resampling is 
performed based on a distribution $F(\hat{\theta})$ known or established, where $\hat{\theta}$ is an estimator of $\theta$. On the other hand, in the nonparametric bootstrap, there is the lack of the distribution $F$. The resampling technique is based on the empirical distribution function $\widehat{F}_{n}$. Resample of $\widehat{F}_{n}$ is equivalent to resample data with replacement.

\subsection{Bootstrap percentile interval}

Let $T_{n}$ be an estimator of the scale $\theta$ based on $n$ observations and $t$ its estimate. Let $T_{n}^{*}$ be the same estimator based on $n$ observations resampling from the original sample with replacement and $t^{*}$ its estimate. For simplicity, suppose $T_{n}$ is a continuous random variable. Denoting the $p$ th quantile of the distribution of the random variable $T_{n}-\theta$ by $a_{p}$, we obtain

$$
\operatorname{Pr}\left\{T_{n}-\theta \leq a_{\frac{\alpha_{1}}{2}}\right\}=\operatorname{Pr}\left\{T_{n}-\theta \geq a_{1-\frac{\alpha_{2}}{2}}\right\}=\frac{\alpha}{2} .
$$

As the amount $Q=T_{n}-\theta$ is invertible and $T_{n}$ depends only on the sample, we can build the confidence interval for $\theta$ rewriting the events above, i.e., we can rewrite the events $T_{n}-\theta \leq a_{\frac{\alpha_{1}}{2}}$ and $T_{n}-\theta \geq a_{1-\frac{\alpha_{2}}{2}}$ with $\theta>T_{n}-a_{\frac{\alpha_{1}}{2}}$ and $\theta<T_{n}-a_{1-\frac{\alpha_{2}}{2}}$, respectively. Thus, the confidence interval of level $\gamma$ is given by the limits

$$
\ell_{\alpha / 2}=t-a_{1-\frac{\alpha_{2}}{2}}, \quad \ell_{1-\alpha / 2}=t-a_{\frac{\alpha_{1}}{2}} .
$$

In many situations, we do not know the distribution of $T_{n}-\theta$. In such cases, suppose that there is some transformation $T_{n}, U=h\left(T_{n}\right)$, such that $U$ has a symmetric distribution. Suppose also that we can obtain the confidence interval of level $1-\alpha$ to $\phi=h(\theta)$. According to Davison and Hinkley (1997), we may use bootstrapping to obtain an approximation to the distribution of $T_{n}-\theta$ using the distribution of $T_{n}^{*}-t$. Thus, we estimate the $p$ th quantile of $T_{n}-\theta$ by the $(J+1) p$ th ordered value of $t^{*}-t$, that is, the $p$ th quantile of $T_{n}-\theta$ is estimated by $t_{((J+1) p)}^{*}-t$. Similarly, the $p$ quantile of $h\left(T_{n}\right)-h(\theta)=U-\phi$ can be estimated by the $(J+1) p$ th ordered value of $h\left(T_{n}^{*}\right)-h(t)=u^{*}-u$. Let $b_{p}$ be the $p$ th quantile of $U-\phi$. Since $U$ has a symmetrical distribution, then $U-\phi$ also has a symmetrical distribution, as soon as it is true that $b_{\frac{\alpha}{2}}=-b_{1-\frac{\alpha}{2}}$. Using the symmetry of $U-\phi$, we have $h\left(\ell_{\alpha / 2}\right)=u+b_{\alpha / 2}$ and $h\left(\ell_{1-\alpha / 2}\right)=u+b_{1-\alpha / 2}$. As $b_{\alpha / 2}$ and $b_{1-\alpha / 2}$ are quantiles of $U-\phi$ and we know how to obtain these quantiles, the lower and upper limits of the confidence intervals are given by $u+\left(u_{((J+1) \alpha / 2)}^{*}-u\right)$ and $u+\left(u_{((J+1)(1-\alpha / 2))}^{*}-u\right)$, respectively, which lead to the limits

$$
u_{((J+1) \alpha / 2),}^{*} \quad u_{((J+1)(1-\alpha / 2))}^{*},
$$

whose transformation to $\theta$ is

$$
t_{(J+1) \alpha / 2}^{*}, \quad t_{(J+1)(1-\alpha / 2)}^{*} .
$$


Note that we do not know the transformation $h$. The confidence interval of level $1-\alpha$ for the parameter $\theta$ does not involve $h$ and it can be evaluated without knowledge of this transformation. The interval (21) is known as the bootstrap interval percentile. According to Davison and Hinkley (1997, p. 203), the percentile method can be applied to any statistic.

\section{Optimization algorithm}

\subsection{Swarm intelligence and particle swarm optimization}

A package developed for $\mathrm{R}$ language very used in the area of continuous distributions is the AdequacyModel package, version 1.0.8, available for download in https://cran.r-project.org/web/packages/AdequacyModel/index.html under the terms of the GPL license $(\geq 2)$.

This package focuses on construction of goodness-of-fit statistics for fitted models and these statistics depend on the MLEs also provided by the package. However, it is noted that several users of the package are having difficulties to obtain these estimates using optimization methods very popular as is the case of NelderMead method (Nelder and Mead, 1965), L-BFGS-B (Nelder and Mead, 1965) and simulated annealing (Belisle, 1992). In general, these users are working with new distributions that have a large number of parameters. The main problem is summed up by the difficulty to obtain initial parameter values for these methods and nonconvergence of the algorithm for global maximization of the log-likelihood function of these models. In this sense, the swarm intelligence proved to be a good strategy for the optimization of these functions and, in general, produce better results and with the advantage of not having to provide initial values. The Swarm Intelligence (SI) is the term used to designate systems of artificial intelligence, where the collective behavior of individuals in a population provides simple solutions or consistent patterns emerge.

The SI was employed using the Particle Swarm Optimization (PSO) method developed by Eberhart and Kennedy (1995) to obtain the MLEs of the model parameters and in the study of simulation about the interval estimates for bootstrap percentile as presented in Section 7.3, as well as to obtain the MLEs for different models considered in applying the ELG-G model (see Section 8). The implementation of the PSO method built in this work will be improved and adapted to later versions of the AdequacyModel package.

Eberhart and Kennedy (1995) developed the PSO method using as a basis the studies of Reynolds (1987) and Heppner and Grenander (1990) that provided models of simulations of flock of birds. The PSO method optimizes a problem by having a population of candidate solutions and moving these particles around in the search-space according to simple mathematical formulae over the particle's position and velocity. The movement of the particles in the search space is randomized. 
The PSO algorithm is presented below, where $f: \mathbb{R}^{n} \mapsto \mathbb{R}$ is the objective function to be minimized, $S$ is the number of particles in the swarm (set of feasible points, i.e., search region), each particle having a vector position $x_{i} \in \mathbb{R}^{n}$ in the search-space and a vector velocity defined by $v_{i} \in \mathbb{R}^{n}$. Let $p_{i}$ be the best known position of particle $i$ and $g$ the best position of all particles.

1. For each particle $i=1, \ldots, S$ do:

- Initialize the particle's position with a uniformly distributed random vector: $x_{i} \sim U\left(b_{l o}, b_{u p}\right)$, where $b_{l o}$ and $b_{u p}$ are the lower and upper boundaries of the search-space.

- Initialize the particle's best known position to its initial position: $p_{i} \longleftrightarrow x_{i}$.

- If $f\left(p_{i}\right)<f(g)$ update the swarm's best known position: $g \leftrightarrow p_{i}$.

- Initialize the particle's velocity: $v_{i} \sim U\left(-\left|b_{u p}-b_{l o}\right|,\left|b_{u p}-b_{l o}\right|\right)$.

2. Until a termination criterion is met (e.g. number of iterations performed, or a solution with adequate objective function value is found), repeat:

- For each particle $i=1, \ldots, S$ do:

- Pick random numbers: $r_{p}, r_{g} \sim U(0,1)$.

- For each dimension $d=1, \ldots, n$ do:

* Update the particle's velocity: $v_{i, d} \leftarrow \omega v_{i, d}+\varphi_{p} r_{p}\left(p_{i, d}-x_{i, d}\right)+$ $\varphi_{g} r_{g}\left(g_{d}-x_{i, d}\right)$.

- Update the particle's position: $x_{i} \leftrightarrow x_{i}+v_{i}$.

- If $f\left(x_{i}\right)<f\left(p_{i}\right)$ do:

* Update the particle's best known position: $p_{i} \leftrightarrow x_{i}$.

* If $f\left(p_{i}\right)<f(g)$ update the swarm's best known position: $g \leftrightarrow p_{i}$.

3. Now $g$ holds the best found solution.

The parameter $\omega$ is called inertia coefficient and as the name implies controls the inertia of each particle arranged in the search region. The quantities $\omega_{p}$ and $\omega_{g}$ control the acceleration of each particle and are called accelerated coefficients. The variance of the best candidates can be used as a stopping criterion, that is, the algorithm will stop if the variance is less than some real $\varepsilon>0$.

\subsection{Hardware used}

The law that established the II PLANIN (Plan Informatics and Automation), approved by the Brazilian National Congress in October 1991, proposed the installation of a National Center for Supercomputing (CESUP) to provide advanced computing services to brazilian researchers. This center was set up at the Federal University of Rio Grande do Sul (UFRGS). The Brazil has some High Performance Processing National Centers (CENAPAD).

We use the hardware available in the National Supercomputing CenterCESUP (CENAPAD UFRGS). The CESUP has two clusters: Cluster Sun Fire, dubbed of Newton, and the Altix cluster SGI, also known as Gauss. The cluster settings are described below. 


\section{Cluster Sun Fire (Newton):}

- 45 processing nodes and 3 nodes of management; 8 GPUs nVIDIA Tesla; 1 GPU AMD FireStream; 1 switch Voltaire InfiniBand; total of 1296 GB of RAM memory; total of $188 \mathrm{~TB}$ of storage capacity, wherein $158 \mathrm{~TB}$ are shared with the cluster SGI Altix Gauss; theoretical performance peak of 12.94 Tflops.

\section{Cluster SGI Altix (Gauss):}

- 64 blades of the processing and 3 nodes of service; total of 4 TB of RAM memory; total of $174 \mathrm{~TB}$ of storage capacity, wherein $158 \mathrm{~TB}$ are shared with the cluster Sun Fire (Newton); theoretical performance peak of 15.97 Tflops.

We use the cluster SGI Altix (Gauss). Each of the 64 units processing of the SGI Altix cluster has 2 dodeca-core processors ( 24 cores) AMD Opteron working at a frequency of $2.3 \mathrm{GHz}, 128 \mathrm{~KB}$ of cache L1 per core (data + instructions), $512 \mathrm{~KB}$ of cache L2 per core and $12 \mathrm{MB}$ of cache L3 per socket. Cluster Gauss has integrated DDR3 memory controller that supports frequencies of $1333 \mathrm{MHz}$ and bandwidth of $42.7 \mathrm{~GB} / \mathrm{s}$ per CPU, totaling $64 \mathrm{~GB}$ of RAM per unit. More information about the hardware available by CESUP can be found in http://www. cesup.ufrgs.br/.

These hardwares provide greater speed in Monte Carlo simulations to be presented later. We use the R programming language and the Rmpi, doSNOW and foreach packages to create the parallel processes. The Rmpi package provides an interface (wrapper) to MPI APIs. It also provides interactive R slave environment. The doSNOW package provides a parallel backend for the $\%$ dopar\% function (function available in the foreach package for parallelization of loops) using the Rmpi package.

\subsection{Simulation study}

We present some Monte Carlo simulations to evaluate the performance of interval estimates by bootstrap percentile. We simulate 20,000 trails of the ELG-Weibull true model with fixed parameters $a=0.5, b=1.5$ and $c=1.5$, with the baseline Weibull distribution having parameters $\alpha=1.5$ and $\beta=1.5$. We take the nominal level of $95 \%$ and sample sizes: 20,60,100 and 500. We consider 500 bootstrap resampling in each Monte Carlo iteration.

The evaluation of the random intervals obtained by bootstrap percentile is taken by the level of coverage of these intervals, that is, we determine the percentage of confidence intervals containing the true parameter within the interval. Taking advantage of the bootstrap used for construction the percentile intervals, we evaluate the standard errors of the MLEs obtained by the PSO method.

Table 1 gives the average of the standard errors obtained using non-parametric bootstrap. This table also provides the averages of the amplitudes of interval estimates, the coverage of the intervals for different sample sizes and the times (in 
Table 1 Percentage of coverage and average amplitude of interval estimates for the parameters added by the generator by bootstrap percentile at a nominal level of $95 \%$

\begin{tabular}{lccccc}
\hline$n$ & Parameter & Coverage (\%) & Amplitude & Error & Time (in hours) \\
\hline \multirow{3}{*}{$n=20$} & $a$ & 86.7854 & 1.3456 & 2.3325 & \\
& $b$ & 87.4416 & 1.7376 & 4.1464 & 100.4356 \\
& $c$ & 89.7584 & 2.1222 & 1.4353 & \\
$n=60$ & $a$ & 91.5443 & 2.1210 & 1.2756 & \\
& $b$ & 90.5432 & 1.5436 & 3.2542 & 170.1102 \\
& $c$ & 92.3453 & 2.0787 & 2.4252 & \\
$n=100$ & $a$ & 93.4545 & 1.5345 & 2.3431 & \\
& $b$ & 92.4539 & 2.4536 & 2.3014 & 213.4464 \\
& $c$ & 94.4235 & 3.3445 & 3.3945 & \\
$n=500$ & $a$ & 93.9716 & 1.5646 & 2.1455 & \\
& $b$ & 94.2325 & 2.5345 & 2.5514 & 432.5643 \\
& $c$ & 95.1215 & 3.2148 & 3.8789 & \\
\hline
\end{tabular}

hours) spent in the of the simulations. For small samples, in particular, for $n=20$, we note that the interval estimates by bootstrap percentiles coverage far below the fixed nominal level. The coverage becomes reasonably close to the $95 \%$ nominal level in large samples (100 and 500).

The amplitudes of interval estimates are small for all scenarios of the simulations. We also compute the bootstrap errors for each iteration of Monte Carlo. We note small errors for all estimates of the ELG-Weibull model. We evaluate the errors by bootstrap because there is no exact way to obtain the errors of the MLEs using the PSO method. Figure 9 displays boxplots of the errors for the parameters added in the ELG-Weibull distribution.

\section{Applications}

In this section, the usefulness of the ELG-Weibull distribution is proved empirically by means of two real data sets. In the applications, we use the AdequacyModel package version 1.0.8 available in the R programming language. First, we consider a data set from Smith and Naylor (1987). The data are the strengths of $1.5 \mathrm{~cm}$ glass fibres, measured at the National Physical Laboratory, England. Unfortunately, the units of measurement are not given in the paper. The data set is also available for download at http://www.stat.ncsu.edu/research/sas/sicl/data/. The second application takes into account the data related to the percentages of body fat determined by underwater weighting and various body circumference measurements for 250 men. For details about the data set, see http://lib.stat.cmu. edu/datasets/. Table 2 gives some descriptive statistics for the two data sets. They are obtained in the AdequacyModel package (version 1.0.8). 


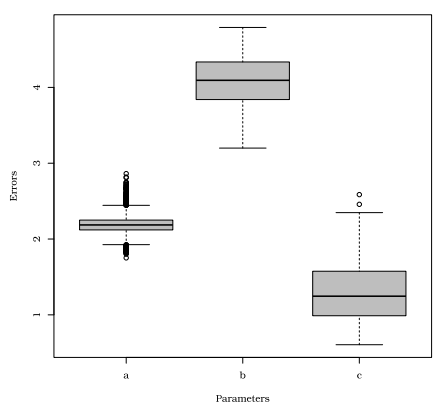

(a) Sample size 20

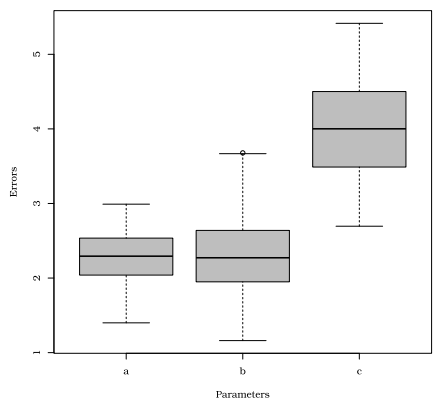

(c) Sample size 100

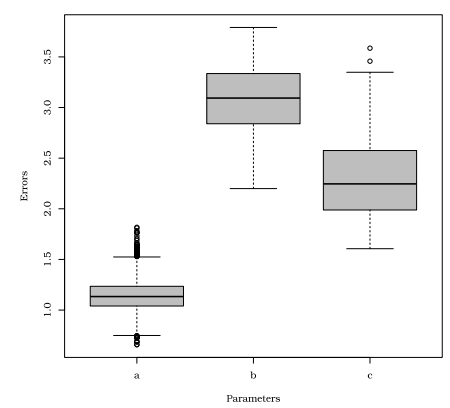

(b) Sample size 60

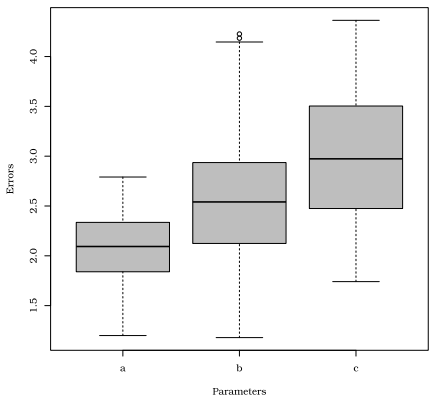

(d) Sample size 500

Figure 9 Errors evaluated by bootstrap and the MLEs obtained by the PSO method with 500 bootstrap replicates.

Table 2 Descriptive statistics

\begin{tabular}{lcc}
\hline & \multicolumn{2}{c}{ Real data sets } \\
\cline { 2 - 3 } Statistics & Glass fibres & \multicolumn{1}{c}{ Body fat $(\%)$} \\
\hline Mean & 1.5068 & 19.3012 \\
Median & 1.5900 & 19.2500 \\
Mode & 1.7000 & 22.5000 \\
Variance & 0.1051 & 67.7355 \\
Skewness & -0.8999 & 0.1953 \\
Kurtosis & 0.9238 & -0.3815 \\
Maximum & 2.2400 & 47.5000 \\
Minimum & 0.5500 & 3.0000 \\
$n$ & 63 & 250 \\
\hline
\end{tabular}




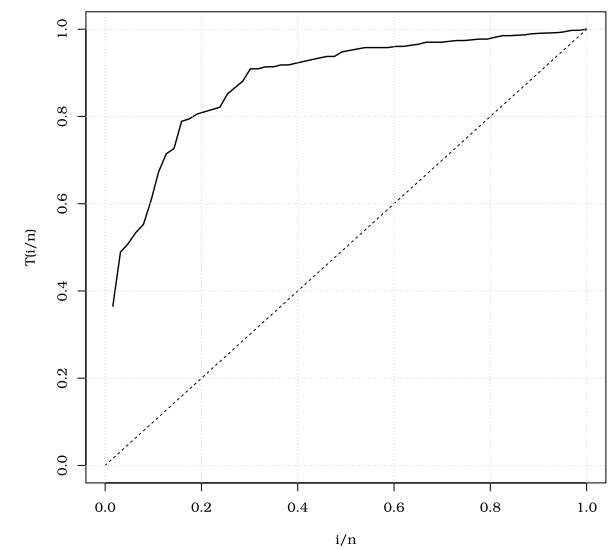

(a)

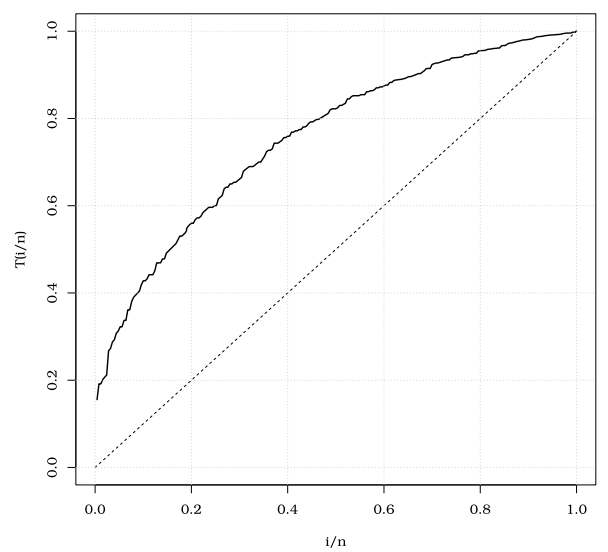

(b)

Figure 10 The TTT plots for the: (a) glass fibres and (b) percentage of body fat.

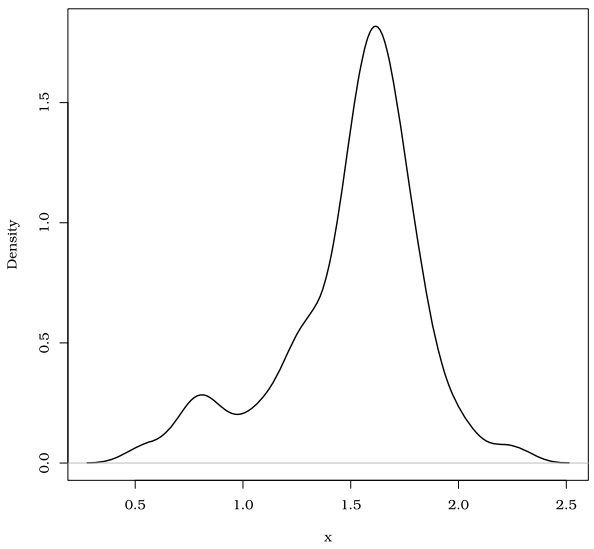

(a)

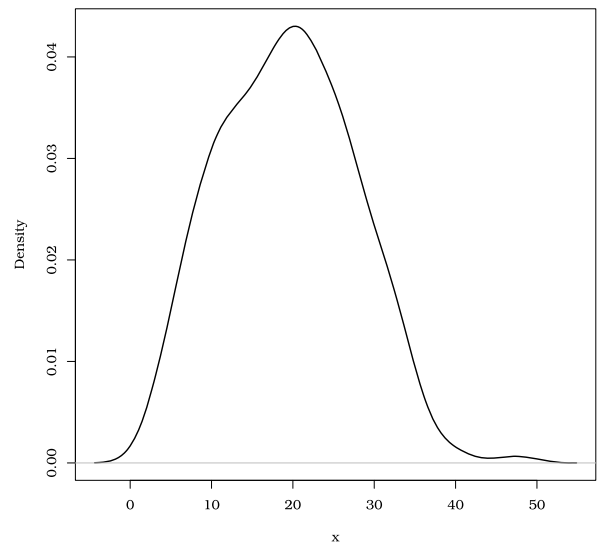

(b)

Figure 11 The Gaussian kernel density estimation for: (a) the glass fibres and (b) percentage of body fat.

One of the important devices, which can help selecting a particular model, is the total time on test (TTT) plot (Aarset, 1987). The TTT plots for the fibres data and for the number of successive failure data are displayed in Figure 10. Both TTT plots in Figure 10 yield a concave curve and then an increasing hrf. Then, these plots indicate the appropriateness of the ELG-Weibull distribution to fit these data. Figure 11 displays the Gaussian kernel density estimation for the glass fibres data and percentage of body fat data.

For these data sets, we fit the ELG-Weibull (ELG-W) distribution defined by (12) and compare it with the Kumaraswamy Weibull Poisson (KW-WP) (Ramos 
et al., 2015), Kumaraswamy Weibull (KW) (Cordeiro, Ortega and Nadarajah, 2010), exponentiated Weibull (EW) (Mudholkar and Srivastava, 1993), New-type Nadarajah-Haghighi (NTNH) (Lemonte, 2013), modified Weibull (MW) (Xie, Tang and Goh, 2002), Chen (Chen, 2000), gamma and Nadarajah-Haghighi (NH) (Nadarajah and Haghighi, 2011) distributions. The MLEs of the model parameters (with standard errors in parentheses) for the ELG-W, Kw-WP, EW, NTNH and other models are listed in Table 3 for the two data sets. The R language is also used to obtain the MLEs by the heuristic method of global optimization (PSO) discussed in the previous section.

We can also perform formal goodness-of-fit tests in order to verify which distribution fits better to these data. We consider the Cramér-von Mises (W) and Anderson-Darling (A), described in details by Chen and Balakrishnan (1995), and Kolmogorov-Smirnov (KS) statistics. In general, the smaller the values of these statistics, the better the fit is. Table 4 gives the values of the Akaike information criterion (AIC), Bayesian information criterion (BIC), consistent Akaike information criterion (CAIC) and Hannan-Quinn information criterion (HQIC), and the A, W and KS statistics for the both models fitted to both data sets. Thus, according to these formal tests, the ELG-W model fits the data sets better than the other distributions. Since the values of the AIC, CAIC and HQIC statistics are smaller for the ELG-W distribution compared to those values of the other fitted models, the new distribution is a very competitive model to explain these data.

Plots of the estimated pdfs, cdfs and survival functions of the ELG-W distribution and other models fitted to both data sets are displayed in Figures 12, 13 and 14, respectively. They reveal that the ELG-W distribution is superior to the other distributions in terms of model fitting.

\section{Conclusions}

We define a new class of distributions called the exponentiated logarithmic generated ("ELG") family. The proposed family can be motivated by compounding the exponentiated generated construction and the logarithmic distribution. It can provide better fits than some well-known lifetime distributions, which represents an interesting property for application. We derive some of its structural properties including moments, quantile and generating functions and order statistics. We use the maximum likelihood method to estimate the model parameters. We provide a simulation study to show the accuracy of the estimates. Further, we adopt the bootstrap percentile technique to obtain confidence intervals for the model parameters. We give two applications to real data to illustrate the potentiality of the proposed family. We hope this generalization may attract wider applications in statistics. 
Table 3 MLEs for the glass fibres data denoted by (I) and percentage of body fat data denoted by (II)

\begin{tabular}{|c|c|c|c|c|c|c|}
\hline \multirow[t]{2}{*}{ Data set } & \multirow{2}{*}{$\frac{\text { Distribution }}{\text { ELG-W }(\alpha, \beta, a, b, c)}$} & \multicolumn{5}{|c|}{ Estimates and standard errors in parenthesis } \\
\hline & & $\begin{array}{c}3.5684 \\
(1.1281)\end{array}$ & $\begin{array}{c}1.1824 \\
(0.1495)\end{array}$ & $\begin{array}{c}0.9944 \\
(2.9934)\end{array}$ & $\begin{array}{c}0.1650 \\
(3.0763)\end{array}$ & $\begin{array}{c}2.7926 \\
(2.0636)\end{array}$ \\
\hline \multirow{7}{*}{ I } & $\mathrm{Kw}-\mathrm{WP}(a, b, c, \lambda, \beta)$ & $\begin{array}{c}0.9377 \\
(4.3973)\end{array}$ & $\begin{array}{c}3.2395 \\
(3.7067)\end{array}$ & $\begin{array}{c}6.4021 \\
(4.0625)\end{array}$ & $\begin{array}{l}17.2578 \\
(0.7777)\end{array}$ & $\begin{array}{c}0.3163 \\
(0.2853)\end{array}$ \\
\hline & $\mathrm{Kw}-\mathrm{W}(a, b, c, \beta)$ & $\begin{array}{c}7.7418 \\
(5.7793)\end{array}$ & $\begin{array}{c}2.6226 \\
(5.2691)\end{array}$ & $\begin{array}{c}0.7262 \\
(6.3079)\end{array}$ & $\begin{array}{l}14.1218 \\
(3.3187)\end{array}$ & \\
\hline & $\mathrm{EW}(\alpha, \beta, a)$ & $\begin{array}{c}7.2846 \\
(4.0527)\end{array}$ & $\begin{array}{c}1.7181 \\
(3.7499)\end{array}$ & $\begin{array}{c}0.6712 \\
(8.2724)\end{array}$ & & \\
\hline & $\mathrm{NTNH}(\alpha, \lambda, \beta)$ & $\begin{array}{c}14.2416 \\
(0.7176)\end{array}$ & $\begin{array}{c}0.0648 \\
(3.0746)\end{array}$ & $\begin{array}{c}9.9259 \\
(2.2890)\end{array}$ & & \\
\hline & $\operatorname{MW}(\alpha, \beta, \lambda)$ & $\begin{array}{c}3.1640 \\
(0.3546)\end{array}$ & $\begin{array}{c}5.6882 \\
(5.1581)\end{array}$ & $\begin{array}{l}13.6191 \\
(2.9185)\end{array}$ & & \\
\hline & Chen $(\lambda, \beta)$ & $\begin{array}{c}0.0720 \\
(0.3563)\end{array}$ & $\begin{array}{c}1.9604 \\
(0.2213)\end{array}$ & & & \\
\hline & $\operatorname{Gamma}(\alpha, \beta)$ & $\begin{array}{c}0.0864 \\
(0.6844)\end{array}$ & $\begin{array}{l}17.4396 \\
(0.4654)\end{array}$ & & & \\
\hline \multirow{10}{*}{ II } & $\mathrm{NH}(\alpha, \lambda)$ & $\begin{array}{c}24.884 \\
(0.1102)\end{array}$ & $\begin{array}{c}0.0212 \\
(0.2334)\end{array}$ & & & \\
\hline & ELG-W $(\alpha, \beta, a, b, c)$ & $\begin{array}{c}1.8303 \\
(0.2172)\end{array}$ & $\begin{array}{l}12.8023 \\
(0.0332)\end{array}$ & $\begin{array}{c}0.9733 \\
(0.1634)\end{array}$ & $\begin{array}{c}2.5701 \\
(5.1669)\end{array}$ & $\begin{array}{c}0.6136 \\
(3.7660)\end{array}$ \\
\hline & $\mathrm{Kw}-\mathrm{WP}(a, b, c, \lambda, \beta)$ & $\begin{array}{l}22.1310 \\
(4.6427)\end{array}$ & $\begin{array}{l}24.9999 \\
(3.4670)\end{array}$ & $\begin{array}{c}0.2568 \\
(0.0530)\end{array}$ & $\begin{array}{l}13.9977 \\
(5.0103)\end{array}$ & $\begin{array}{c}0.2074 \\
(6.9024)\end{array}$ \\
\hline & $\mathrm{Kw}-\mathrm{W}(a, b, c, \beta)$ & $\begin{array}{c}0.5013 \\
(3.7177)\end{array}$ & $\begin{array}{l}14.8283 \\
(6.5498)\end{array}$ & $\begin{array}{c}8.9928 \\
(1.6592)\end{array}$ & $\begin{array}{l}24.9999 \\
(1.5868)\end{array}$ & \\
\hline & $\mathrm{EW}(\alpha, \beta, a)$ & $\begin{array}{c}3.0043 \\
(0.2875)\end{array}$ & $\begin{array}{l}23.8105 \\
(1.1316)\end{array}$ & $\begin{array}{c}0.7558 \\
(3.3796)\end{array}$ & & \\
\hline & $\mathrm{NTNH}(\alpha, \lambda, \beta)$ & $\begin{array}{c}1.8273 \\
(4.9998)\end{array}$ & $\begin{array}{c}0.0419 \\
(4.0430)\end{array}$ & $\begin{array}{c}3.7999 \\
(6.2450)\end{array}$ & & \\
\hline & $\operatorname{MW}(\alpha, \beta, \lambda)$ & $\begin{array}{l}21.6646 \\
(3.3369)\end{array}$ & $\begin{array}{c}1.5020 \\
(3.4177)\end{array}$ & $\begin{array}{c}0.0241 \\
(6.5183)\end{array}$ & & \\
\hline & Chen $(\lambda, \beta)$ & $\begin{array}{c}0.0065 \\
(2.1056)\end{array}$ & $\begin{array}{c}0.5192 \\
(0.6374)\end{array}$ & & & \\
\hline & $\operatorname{Gamma}(\alpha, \beta)$ & $\begin{array}{c}4.1876 \\
(1.2548)\end{array}$ & $\begin{array}{c}4.6091 \\
(1.0493)\end{array}$ & & & \\
\hline & $\mathrm{NH}(\alpha, \lambda)$ & $\begin{array}{l}17.3791 \\
(0.7384)\end{array}$ & $\begin{array}{c}0.0022 \\
(1.5522)\end{array}$ & & & \\
\hline
\end{tabular}

It is used rounding to the fourth decimal place. 
Table 4 Goodness-of-fit statistics for the glass fibre data denoted by (I) and percentages of body fat data denoted by (II)

\begin{tabular}{|c|c|c|c|c|c|c|c|c|}
\hline Data set & Distribution & A & $\mathrm{W}$ & KS & AIC & CAIC & $\mathrm{BIC}$ & HQIC \\
\hline \multirow{9}{*}{ I } & ELG-W $(\alpha, \beta, a, b, c)$ & 0.5513 & 0.0959 & 0.1105 & 34.4784 & 35.5311 & 45.1941 & 38.6930 \\
\hline & $\mathrm{Kw}-\mathrm{WP}(a, b, c, \lambda, \beta)$ & 1.7612 & 0.3216 & 0.1689 & 44.3267 & 45.3794 & 55.0424 & 48.5413 \\
\hline & $\mathrm{Kw}-\mathrm{W}(a, b, c, \beta)$ & 1.2689 & 0.2304 & 0.1516 & 38.2397 & 38.9293 & 46.8122 & 41.6113 \\
\hline & $\mathrm{EW}(\alpha, \beta, a)$ & 1.1118 & 0.2000 & 0.1462 & 35.3510 & 35.7578 & 41.7804 & 37.8798 \\
\hline & $\operatorname{NTNH}(\alpha, \lambda, \beta)$ & 2.7480 & 0.5006 & 0.2105 & 50.0737 & 50.4805 & 56.5031 & 52.6024 \\
\hline & $\operatorname{MW}(\alpha, \beta, \lambda)$ & 1.2901 & 0.2345 & 0.1514 & 36.3860 & 36.7927 & 42.8154 & 38.9147 \\
\hline & Chen $(\lambda, \beta)$ & 0.9623 & 0.1615 & 0.1373 & 36.9227 & 37.1227 & 41.2090 & 38.6085 \\
\hline & $\operatorname{Gamma}(\alpha, \beta)$ & 3.1174 & 0.5684 & 0.2164 & 51.9031 & 52.1031 & 56.1893 & 53.5889 \\
\hline & $\mathrm{NH}(\alpha, \lambda)$ & 2.3541 & 0.4294 & 0.4513 & 143.1259 & 143.3259 & 147.4121 & 144.8117 \\
\hline \multirow{9}{*}{ II } & ELG-W $(\alpha, \beta, a, b, c)$ & 0.1246 & 0.0164 & 0.0242 & 1758.6590 & 1758.9050 & 1776.2660 & 1765.7460 \\
\hline & $\mathrm{Kw}-\mathrm{WP}(a, b, c, \lambda, \beta)$ & 1.3448 & 0.2200 & 0.0627 & 1772.8220 & 1773.0680 & 1790.4290 & 1779.9080 \\
\hline & $\mathrm{Kw}-\mathrm{W}(a, b, c, \beta)$ & 1.3420 & 0.2204 & 0.0667 & 1770.3700 & 1770.5330 & 1784.4560 & 1776.0390 \\
\hline & $\mathrm{EW}(\alpha, \beta, a)$ & 0.2549 & 0.0347 & 0.0327 & 1757.7540 & 1757.8510 & 1768.3180 & 1762.0050 \\
\hline & $\operatorname{NTNH}(\alpha, \lambda, \beta)$ & 1.6362 & 0.2702 & 0.0743 & 1771.6980 & 1771.7950 & 1782.2620 & 1775.9490 \\
\hline & $\operatorname{MW}(\alpha, \beta, \lambda)$ & 0.4229 & 0.0525 & 0.0399 & 1771.6800 & 1771.7770 & 1782.2440 & 1775.9310 \\
\hline & Chen $(\lambda, \beta)$ & 0.5479 & 0.0688 & 0.0360 & 1768.4090 & 1768.4580 & 1775.4520 & 1771.2440 \\
\hline & $\operatorname{Gamma}(\alpha, \beta)$ & 1.9585 & 0.3240 & 0.0764 & 1773.3760 & 1773.4240 & 1780.4180 & 1776.2100 \\
\hline & $\mathrm{NH}(\alpha, \lambda)$ & 0.4922 & 0.0772 & 0.2186 & 1872.0240 & 1872.0730 & 1879.0670 & 1874.8590 \\
\hline
\end{tabular}

1 - The statistics are obtained in the AdequacyModel package, version 1.0.8.

2 - It is used rounding to the fourth decimal place. 


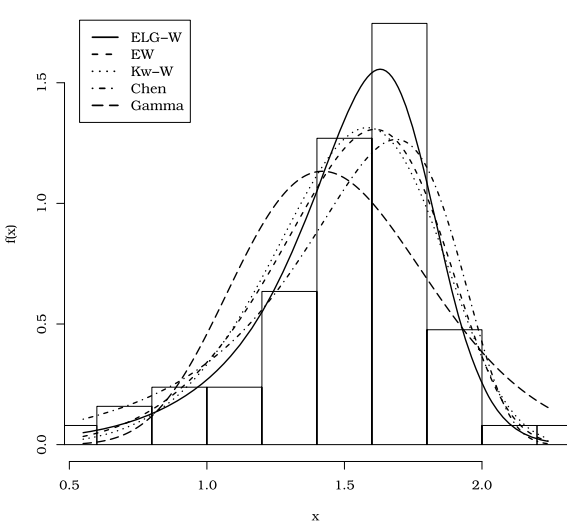

(a)

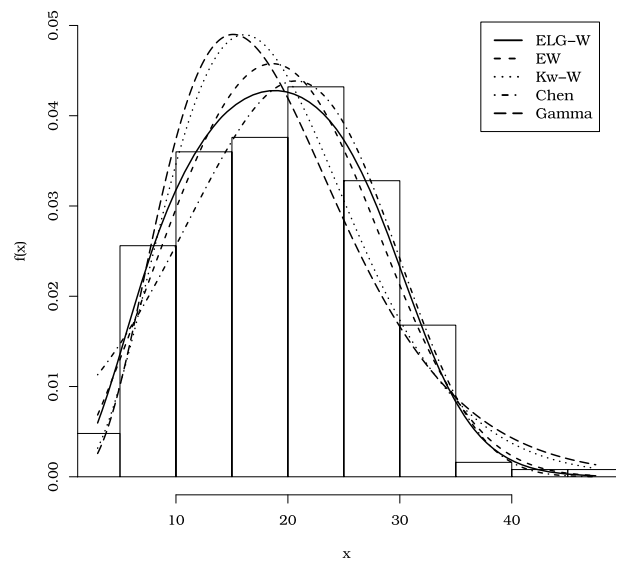

(b)

Figure 12 Estimates of the density functions for the: (a) glass fibres (b) percentages of body fat.

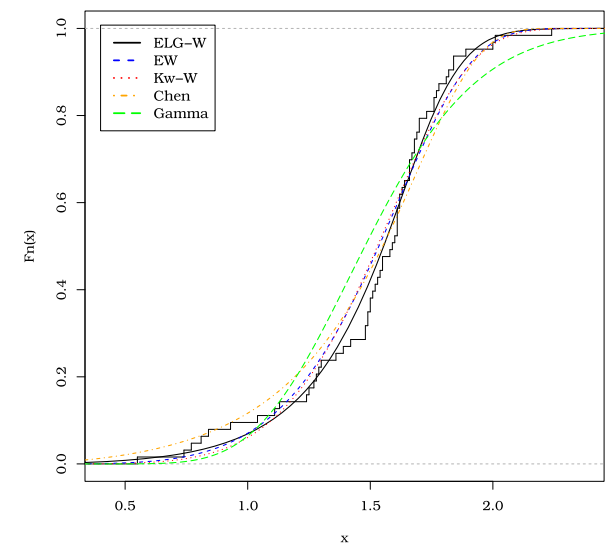

(a)

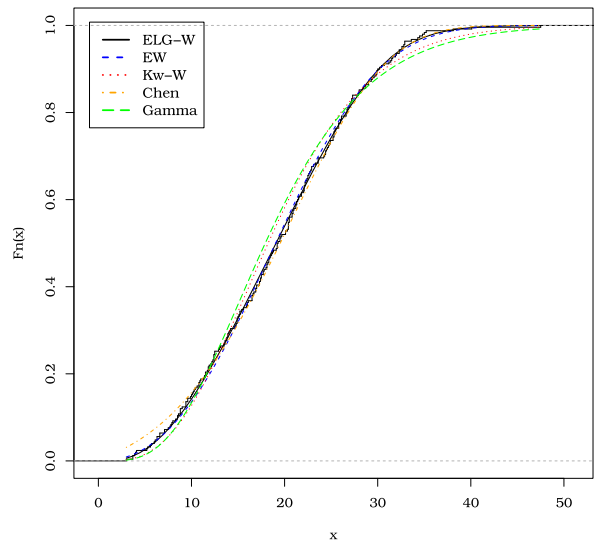

(b)

Figure 13 Estimates of the distribution functions and empirical distribution for the: (a) glass fibre and (b) percentages of body fat.

\section{Appendix: Script in Julia language}

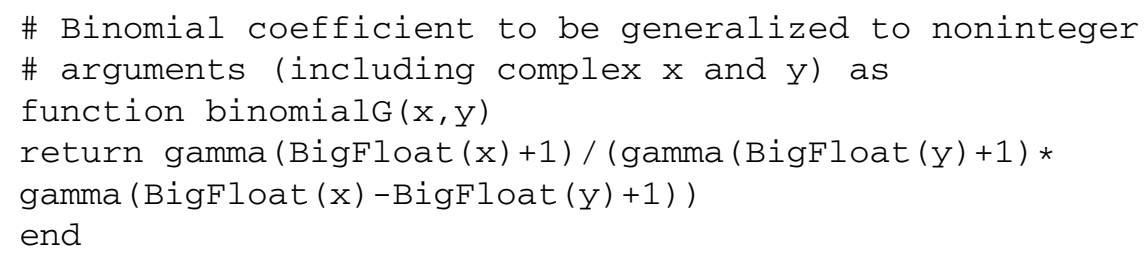


P. R. D. Marinho et al.

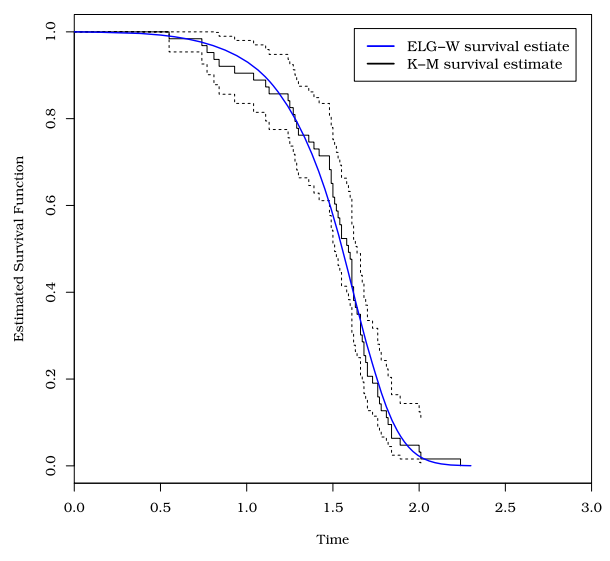

(a)

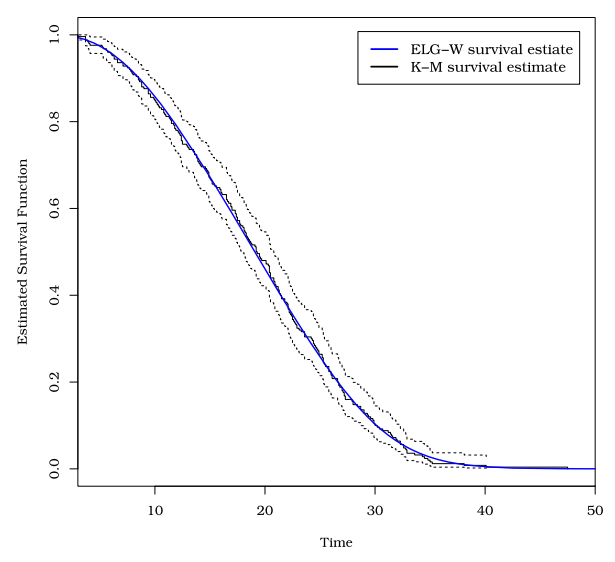

(b)

Figure 14 Kaplan-Meier (K-M) estimates compared with the ELG-W survival estimates for the: (a) glass fibres and (b) percentages of body fat.

\# Stirling numbers of the second kind.

function $\mathrm{S}(\mathrm{n}, \mathrm{m})$

$\mathrm{a}=1 / \operatorname{gamma}(\mathrm{m}+1)$

$\mathrm{v}=\operatorname{zeros}(\mathrm{Float} 64, \operatorname{int}(\operatorname{trunc}(\mathrm{m}))+1,1)$

for $i=0:($ length $(v)-1)$

$v[i+1]=(-1)^{\wedge} i * \operatorname{binomialg}(m, i) *(m-i) \wedge n$

end

return $a * \operatorname{sum}(v)$

end

\# Stirling numbers of the first kind.

function $s(n, m)$

if $n<m$

return 0

else

$\mathrm{V}=\operatorname{zeros}($ Float64, int $(\operatorname{trunc}(n-m))+1,1)$

for $\mathrm{k}=0:($ length $(\mathrm{v})-1)$

$\mathrm{v}[\mathrm{k}+1]=(-1) \wedge \mathrm{k} * \mathrm{binomialG}(\mathrm{k}+\mathrm{n}-1, \mathrm{k}+\mathrm{n}-\mathrm{m})$ *

binomialG $(2 * n-m, n-k-m) * S(k-m+n, k)$

end

return sum $(v)$

end

end

\# Stirling polynomial defined in

\# http://mathworld.wolfram.com/StirlingPolynomial.html

function $\operatorname{poly}(\mathrm{n}, \mathrm{m})$ 


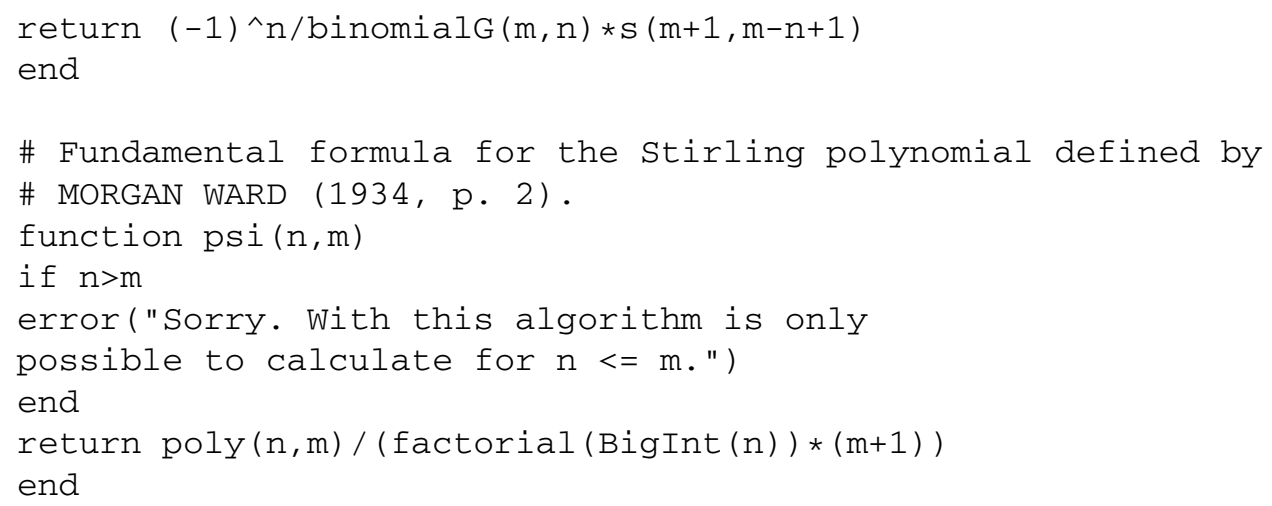

\section{References}

Aarset, M. V. (1987). How to identify bathtub hazard rate. IEEE Transactions on Reliability 36, 106-108.

Belisle, C. J. P. (1992). Convergence theorems for a class of simulated annealing algorithms on $\mathbb{R}^{d}$. Journal of Applied Probability 29, 885-895. MR1188544

Bezanson, J., Karpinski, S., Shah, V. B. and Edelman, A. (2012). Julia: A fast dynamic language for technical computing. Preprint. Available at arXiv:1209.5145.

Castellares, F. and Lemonte, A. J. (2015). A new generalized Weibull distribution generated by gamma random variables. Journal of the Egyptian Mathematical Society 23, 382-390. MR3355432

Chen, Z. (2000). A new two-parameter lifetime distribution with bathtub shape or increasing failure rate function. Statistics and Probability Letters 49, 155-161. MR1790165

Chen, G. and Balakrishnan, N. (1995). A general purpose approximate goodness-of-fit test. Journal of Quality Technology 27, 154-161.

Cordeiro, G. M. and de Castro, M. (2011). A new family of generalized distributions. Journal of Statistical Computation and Simulation 81, 883-898. MR2806932

Cordeiro, G. M., Ortega, E. M. M. and Nadarajah, S. (2010). The Kumaraswamy Weibull distribution with application to failure data. Journal of the Franklin Institute 347, 1399-1429. MR2720934

Davison, A. and Hinkley, D. (1997). Bootstrap Methods and Their Application. Cambridge: Cambridge University Press. MR1478673

Eberhart, R. C. and Kennedy, J. (1995). A new optimizer using particle swarm theory. In Proceedings of the Sixth International Symposium on Micro Machine and Human Science, Vol. 1, 39-43.

Efron, B. (1979). Bootstrap methods: Another look at the Jackknife. The Annals of Statistics 7, 1-26. MR0515681

Eugene, N., Lee, C. and Famoye, F. (2002). Beta-normal distribution and its applications. Communications in Statistics-Theory and Methods 31, 497-512. MR1902307

Flajonet, P. and Odlyzko, A. (1990). Singularity analysis of generating function. SIAM Journal on Discrete Mathematics 3, 216-240. MR1039294

Flajonet, P. and Sedgewick, R. (2009). Analytic Combinatorics. Cambridge: Cambridge University Press. MR2483235

Heppner, F. and Grenander, U. (1990). A stochastic nonlinear model for coordinated bird flocks. In The Ubiquity of Chaos, 233-238.

Lemonte, J. A. (2013). A new exponential-type distribution with constant, decreasing, increasing, upside-down bathtub and bathtub-shaped failure rate function. Computational Statistics and Data Analysis 62, 149-170. MR3040269 
Mudholkar, G. S. and Srivastava, D. K. (1993). Exponentiated Weibull family for analyzing bathtub failure-rate data. IEEE Transactions on Reliability 42, 299-302.

Nadarajah, S. and Haghighi, F. (2011). An extension of the exponential distribution. Statistics 45, 543-558. MR2847341

Nelder, J. A. and Mead, R. (1965). A simplex algorithm for function minimization. Computer Journal 7, 308-313. MR3363409

Ramos, M. W. A., Marinho, P. R. D., Cordeiro, G. M., da Silva, R. V. and Hamedani, G. G. (2015). The Kumaraswamy-G Poisson family of distributions. Journal of Statistical Theory and Applications 14, 222-239. MR3413398

Reynolds, C. W. (1987). Flocks, herds and schools: A distributed behavioral model. ACM Siggraph Computer Graphics 21, 25-34.

Roman, S. (1984). The Umbral Calculus, 59-63. New York: Academic Press. MR0741185

Smith, R. L. and Naylor, J. C. (1987). A comparison of maximum likelihood and Bayesian estimators for the three-parameter Weibull distribution. Applied Statistics 36, 358-369. MR0918854

Ward, M. (1934). The representation of Stirling's numbers and Stirling's polynomials as sums of factorial. American Journal of Mathematics 56, 87-95. MR1507004

Xie, M., Tang, Y. and Goh, T. N. (2002). A modified Weibull extension with bathtub-shaped failure rate function. Reliability Engineering and System Safety 76, 279-285.

Zografos, K. and Balakrishnan, N. (2009). On families of beta- and generalized gamma-generated distributions and associated inference. Statistical Methodology 6, 344-362. MR2751078

P. R. D. Marinho

Department of Statistics

Federal University of Paraíba

João Pessoa

Brazil

E-mail: pedro@de.ufpb.br

F. Peña Ramírez

Department of Statistics

Federal University of Parnambuco

Recife

Brazil

E-mail: fapenara@gmail.com
G. M. Cordeiro

Department of Statistics

Federal University of Parnambuco

Recife

Brazil

E-mail: gauss@de.ufpe.br

M. Alizadeh

Department of Statistics

Persian Gulf University of Bushehr

Bushehr

Iran

E-mail: moradalizadeh78@gmail.com

\author{
M. Bourguignon \\ Department of Statistics \\ Federal University of Rio Grande do Norte \\ Natal \\ Brazil \\ E-mail: m.p.bourguignon@gmail.com
}

